\title{
QUANTITATIVE REPRESENTATION OF THREE-DIMENSIONAL CELL CULTURE MODELS
}

\author{
Hang Chang ${ }^{12}$, Catherine Park ${ }^{1}$, and Bahram Parvin ${ }^{1}$ \\ ${ }^{1}$ Lawrence Berkeley National Laboratory, Berkeley, CA 94720 \\ ${ }^{2}$ Institue of Automation, Chinese Academy of Sciences, Beijng, China
}

\begin{abstract}
Three-dimensional mammary cell culture models offer new opportunities for the development of computational techniques for segmentation, localization, and multicellular organization. Under normal conditions, these assays form a symmetrical, hollow structure, which is necessary for their functional operation. Often, the nuclear compartments are labeled, which provides context for quantitative protein localization or colony structure through fluorescent microscopy. These colonies are first delineated from the background using the level set method. Within each colony, nuclear regions are then bounded by their center of mass through iterative radial voting, and a local neighborhood for each nucleus is established through Voronoi tessellation. Finally, the level set method is applied again within each Voronoi region to delineate the nuclear compartment. The paper concludes with the application of the proposed method to a set of experimental data demonstrating a stable solution when iterative radial voting and level set methods are used synergistically. Furthermore, segmented colonies are characterized for architectural changes as as a result of ionizing radiation.
\end{abstract}

\section{INTRODUCTION}

Current protocol for most biological imaging assays is limited to monolayer cell culture models; however, in the body, cells exist in more complex three-dimensional arrangements, in intimate association with each other and components of their microenvironment. These arrangements are critical to the function and maintenance of the differentiated state. The primary rationale for extending a subset of these protocols to $3 \mathrm{D}$ cell culture models is that they provide much more faithful replicates of cell behavior in vivo than is possible using 2D substrata. While the information these cultures can provide is undoubtedly more valuable, the experiments are much harder to set up, and require more advanced quantitative tools for phenotypic characterization. Furthermore, efficient and robust computational requirements for these experiments have been a rate-limiting issue due to a more complex phenotypic signature. A sample of these 3D colonies, at one focal plane, is shown in Figure 1. For a certain class of studies, three-dimensional cell culture models fill a gap between monolayer and in vivo models [5]. Although the former may be appropriate as an initial step toward discovery and for certain aspects of biological studies, the later is more expensive and timeconsuming, and as a result cannot scale for high-throughput studies targeting different end points. However, 3D assays introduce significant computational challenges: (i) subcellular compartments often

THE RESEARCH WAS SUPPORTED BY THE MEDICAL IMAGING PROGRAM, OFFICE OF BIOLOGICAL EFFECTS RESEARCH, U.S. DEPARTMENT OF ENERGY, AND NATIONAL CANCER INSTITUTE UNDER GRANT NO. DE-FG03-01ER63240. PUBID IS LBNL-62470. AUTHORS THANK VANDERBILT UNIVERSITY FOR PROVIDING SOME OF THE DATA FOR THIS RESEARCH. overlap, (ii) staining/labeling may not be uniform, and (iii) the scale of labeled compartments may have a large variation.
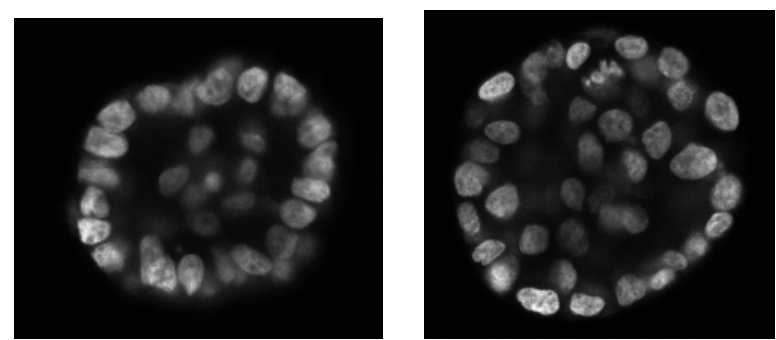

Fig. 1. A slice of a three-dimensional cell culture assay indicating variations in size and intensity and overlapping compartments.

The first step in most biological imaging assays is to label a subcellular compartment (e.g., nuclear) and to provide context for quantifying protein localization. Labeling the subcellular compartment corresponds to segmentation. Research in the segmentation of subcellular structures spans from traditional ad hoc methods of modeling intensity distribution to geometric techniques [3, 4] and surface evolution methods. A key observation is that nuclear regions are often convex and form positive curvature maxima when they overlap each other. This feature was used earlier in 2D segmentation of nuclear regions [4]. However, this method is only applicable to monolayer cell model systems where background is clearly delineated. Our approach is based on constraining the solution to provide seeds corresponding to the nuclear regions and then breaking up local regions based on additional intensity and geometric constraints. The seeding is based on radial voting, where gradient information is projected inward to infer a local center of mass. Once these seeds are established, Voronoi tessellation provides the local neighborhood where each nucleus resides. This local neighborhood is further partitioned based on its intensity distribution using level set methods. Finally, the method is applied to a data set corresponding to samples that are fixed at specific time following ionizing radiation. Segmented nuclei provide the basis for deriving an index for changes in the colony architecture. This index is quantified and tracked at different time points.

Organization of the paper is as follows. Section 2 summarizes previous methods. Section 3 provides a detailed description of the proposed method. Section 4 provides examples and results on the application of the proposed methods on real data. Section 5 concludes the paper.

\section{PREVIOUS RESEARCH}

The difficulties in localization of subcellular compartments are often due to variations in scale, noise, and topology. Other complexities originate from missing data and perceptual boundaries between neighboring compartments. Previous methods for extraction 
of nuclear compartments have relied on either global thresholding or adaptive (localized) thresholding followed by the watershed method for separating adjacent regions. Other methods have relied on modelbased techniques leveraging inherent geometric properties for subcellular compartments $[3,4]$. These methods rely on the notion that the nuclear regions are often convex and, when adjacent compartments overlap, they form folds corresponding to curvature maxima, which can be used for grouping and partitioning.

While geometric methods offer robust model-based constraints for bounding the solution to the segmentation problem, level set methods offer numerically stable solutions to the active contour problems. By limiting and bounding the active contour model to a small nuclear region and its immediate background, a more stable solution is produced, which ensures that (1) overlapping compartments are not merged together, (2) potential leakage in the curve evolution is localized to a small neighborhood, and (3) an improved segmentation can be generated as a result of localized statistics of the region bounding one nucleus.

\section{APPROACH}

Specific steps in delineation of nuclei in a mammosphere system are shown in Figure 2. With the 2D image as an input, the colonies are first segmented by applying the active contour model. Then, within each colony, the location of the centroid of each nucleus is estimated through iterative radial voting. With the estimated seeds for nuclei, each nucleus is then bounded in a Voronoi tessellation, which is further refined by active contour evolution.

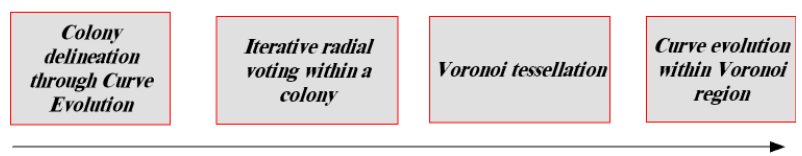

Fig. 2. Steps in delineation of nuclear regions in a mammosphere structure.

\subsection{Colony segmentation with the active contour model}

The active contour model has been used extensively in the medical and biological imaging communities, and many variations of it are currently supported through ITK, from Kitware, Inc. The level set formulation of the active contour model offers a natural and numerically robust implementation of curve evolution equations within the Eulerian formulation. The ITK image library offers several 2D and 3D implementations of curve evolution within the level set framework. The most basic model includes a constant external motion coupled with geometric smoothing. This model essentially behaves like a region-growing method, subject to the continuity of evolving contours while simultaneously handling topological changes. Another model within the ITK library, known as geodesic active contours [1], aims to unify parametric and level set geometric models. This approach formulates the level set concept within an optimization framework. In addition, the ITK library includes a number of level set formulations of active contours that incorporate prior shape models, which can facilitate segmentation of known patterns. Our implementation corresponds to the Mumford-Shah function as modeled by Chan and Vese [2] to segment objects whose boundaries are not necessarily defined by a gradient, thus reducing the leakage experienced by some gradient-based curve evolution functions. The deriving energy functions are governed by forces that are computed from the interior and exterior of evolving boundaries. A brief
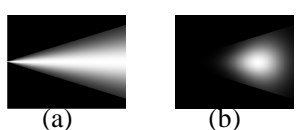

Fig. 3. Kernel topography: (a-e) Evolving kernel for the detection of radial symmetries (shown at a fixed orientation) has a trapezoidal active area with Gaussian distribution along both axes.

overview follows. The energy functional $F\left(c_{1}, c_{2}, C\right)$ is defined by

$$
\begin{aligned}
F\left(c_{1}, c_{2}, C\right) & =\mu \cdot \text { Length }(C) \\
& +v \cdot \text { Area }(\text { inside }(C)) \\
& +\lambda_{1} \int_{\text {inside }(C)}\left|u_{0}(x, y)-c_{1}\right|^{2} d x d y \\
& +\lambda_{2} \int_{\text {outside }(C)}\left|u_{0}(x, y)-c_{2}\right|^{2} d x d y
\end{aligned}
$$

where $u_{0}$ corresponds to the image, $c_{1}$ and $c_{2}$ are the foreground and background intensity, and $\mu \geq 0, v \geq 0, \lambda_{1}, \lambda_{2} \geq 0$ are fixed parameters. The level set formulation of this model is given by considering $C \subset \Omega$ as the zero level set of a Lipschitz function $\phi: \Omega \rightarrow \mathbb{R}$, in which $\Omega$ is a bounded open subset of $\mathbb{R}^{2}$. Using the Heaviside function $H$, and the one-dimensional Dirac measure $\delta_{0}$, defined by

$$
H(z)=\begin{aligned}
& 1, \quad \text { if } z \geq 0 \\
& 0, \quad \text { if } z<0
\end{aligned} \quad \delta_{0}(z)=\frac{d H(z)}{d z}
$$

The energy form can now be written as

$$
\begin{aligned}
F_{\epsilon}\left(c_{1}, c_{2}, \phi\right) & =\mu \int \delta_{\epsilon}(\phi(x, y))|\nabla \phi(x, y)| d x d y \\
& +v \int H_{\epsilon}(\phi(x, y)) d x d y \\
& +\lambda_{1} \int\left|u_{0}(x, y)-c_{1}\right|^{2} H_{\epsilon}(\phi(x, y)) d x d y \\
& +\lambda_{2} \int\left|u_{0}(x, y)-c_{2}\right|^{2}\left(1-H_{\epsilon}(\phi(x, y))\right) d x d y
\end{aligned}
$$

If $\phi$ is fixed then $c_{1}$ and $c_{2}$ can be expressed as:

$$
\begin{aligned}
c_{1}(\phi) & =\frac{\int_{\Omega} u_{0}(x, y) H_{\epsilon}(\phi(x, y)) d x d y}{\int_{\Omega} H_{\epsilon}(\phi(x, y)) d x d y} \\
c_{2}(\phi) & =\frac{\int_{\Omega} u_{0}(x, y)\left(1-H_{\epsilon}(\phi(x, y))\right) d x d y}{\int_{\Omega}\left(1-H_{\epsilon}(\phi(x, y))\right) d x d y}
\end{aligned}
$$

And by keeping $c_{1}$ and $c_{2}$ fixed, the Euler-Lagrange equation for $\phi$ can be written as

$$
\frac{\partial \phi}{\partial t}=\delta_{\epsilon} \quad \mu d i v \quad \frac{\nabla \phi}{|\nabla \phi|}-v-\lambda_{1}\left(u_{0}-c_{1}\right)^{2}+\lambda_{2}\left(u_{0}-c_{2}\right)^{2}
$$

In our implementation, we have used the regularization of $H$, introduced by [2]:

$$
H_{2, \epsilon}(z)=\frac{1}{2} \quad 1+\frac{2}{\pi} \arctan \frac{z}{\epsilon}
$$

While in some cases thresholding may be sufficient for colony segmentation, we have opted to use the active contour model since it is fast (about 1.8 seconds for an image of 512-by-512 with 5 colonies), avoids ad hoc parameter setting, and will be reused for nuclear segmentation. Examples are included in a later section.

\subsection{Nuclear detection with iterative scalar voting}

Nuclear regions are convex and often radially symmetric, as shown in Figure 1. Complexities arise as a result of nonuniform staining, overlapping nuclei, and variations in size. The underlying theme is to model the nuclear detection through perceptual grouping, thus 


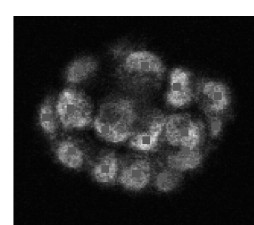

(a)

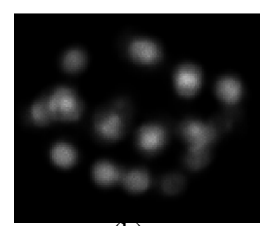

(b)

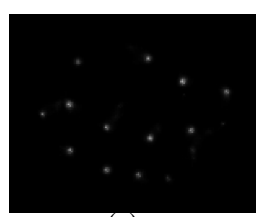

(c)
Fig. 4. Detection of radial symmetries for a mammosphere with multiple overlapping nuclei: (a) original image; and (b-c) voting landscape at several intermediate steps, indicating convergence to a localized region.

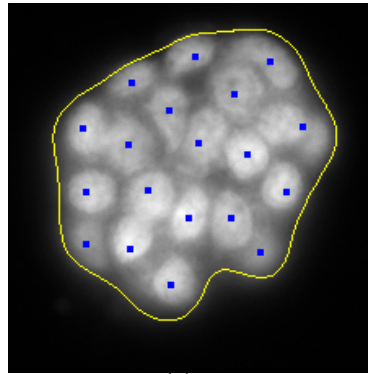

(a)

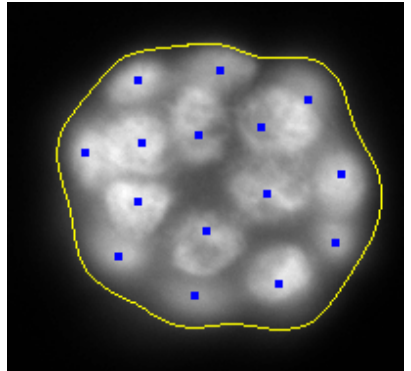

(b)
Fig. 5. Examples of voted nuclei from two mammospheres indicate robust performance in the presence of variation in size and contrast.

making the detection more invariant to noise and variation in size. Voting along the gradient direction provides a hypothesis profile for saliency, i.e. an approximate center of mass. The voting kernel is designed in a way that encodes for a specific saliency and is refined at each iteration step. The shape and evolution of these kernels, inferring center of mass, is shown in Figure 3. Detection of radial symmetry is iterative, where gradient magnitude is projected along the radial direction according to a kernel function. The kernel function is smooth, and its topography becomes more focused and dense at each consecutive iteration, as shown in Figure 3. The details of the algorithm can be found in earlier papers. To illustrate the behavior of iterative voting, Figure 4 shows intermediate steps that lead toward final results for overlapping 2D objects that are generated synthetically. The voting landscape corresponds to the spatial clustering that is initially diffuse and is subsequently refined and focused into distinct regions. Two examples of 2D voting are shown in Figure 5, where each nucleus in a mammosphere has been detected.

\subsection{Nuclear segmentation}

The voting results are used as vertices to generate a local neighborhood through Voronoi tessellation, as shown in Figures 6a-b. This local neighborhood is then used to constrain the nuclear segmentation within a small region. The actual nuclear segmentation is performed with the active contour model described earlier [2], and two results are shown in Figures 6c-d.

\section{EXPERIMENTAL RESULTS}

Ductal carcinoma in situ (DCIS) is characterized by abnormally proliferating lumenal epithelial cells that exhibit a range of changes characteristic of dysregulated cancerous cells, but are contained within the normal boundaries of the breast duct, surrounded by an intact basement membrane. To emulate DCIS in the 3D culture model, we express a constitutively activated myristoylated Akt1 construct into MCF-10A cells. A fundamental question that arises is how

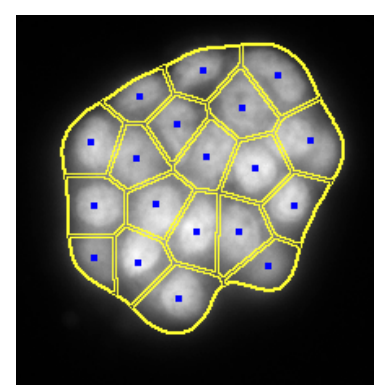

(a)

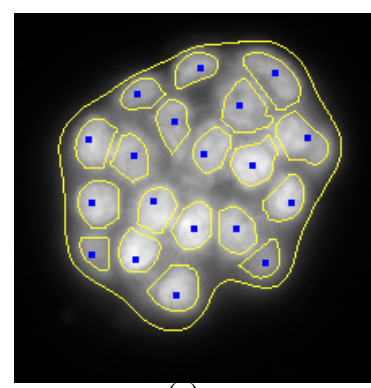

(c)

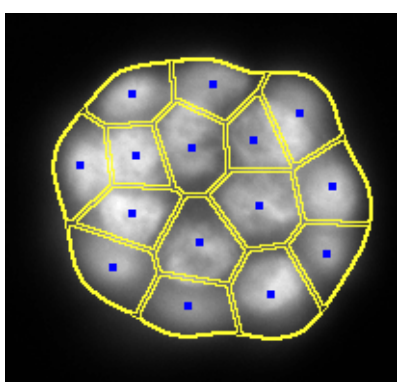

(b)

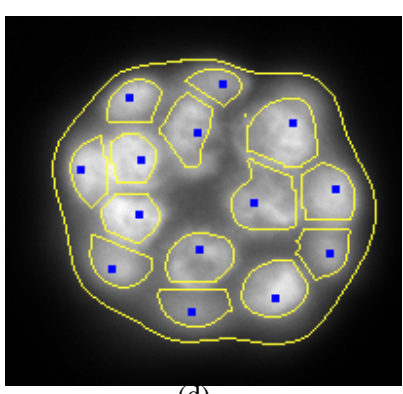

(d)
Fig. 6. Steps in refined segmentation: (a-b) Voronoi tessellation of voted regions, (c-d) refined segmentation within Voronoi regions.

does morphology and structure of the colony is influenced by IR response? An experiment was designed, 74 samples were irradiated, a total of 152 colonies were imaged, and their architecture was quantified using the method described in this paper. On average, each colony consisted of 16 cells, and the segmentation algorithm had a $5 \%$ error in delineation due to excessive overlap between adjacent nuclei and nonuniform staining of nuclear regions. Figure 7 shows several experimental results corresponding to noisy images, overlapping subcellular compartments, and variation in intensities. Nuclear detection through the voting method indicates high confidence, with only one cell missed in the second image. This is due to the fact that only a small part of the cell is visible at this focal plane. The voting results serve as vertices to initiate Voronoi tessellation and a more detailed segmentation of the nuclear region. In the absence of voting and tessellation, nuclear segmentation within the colony is shown in Figure 8 for comparative analysis. Figure 9 shows an example of the entire process from colony delineation to nuclear segmentation. Finally, an experiment was designed to examine the structure of the colonies following an initial treatment as a function of time. Considering that the normal structure of a colony is a hollow sphere, an elliptical model is used to represent the deviation from normality. Highly disorganized colonies have a large error fit, while normal colonies behave in an opposite fashion. Figure 10 shows the result of an ellipse fit for one colony and the trend in colony organization as a function of time. In this experiment, samples were treated with radiation in the presence of a specific concentration of an anticancer drug at time zero, then fixed at 6 hours, 12 hours, and 4 days following the treatment. The plot indicates that colonies become more organized following treatment as a function of time.

\section{CONCLUSION}

We have shown that traditional active contours are not appropriate for segmentation of nuclear regions; however, by constraining the active contour model to a local region, an improved solution can be realized. These local regions are estimated based on the fact that the nuclear regions are round and an estimate to their centers of mass 

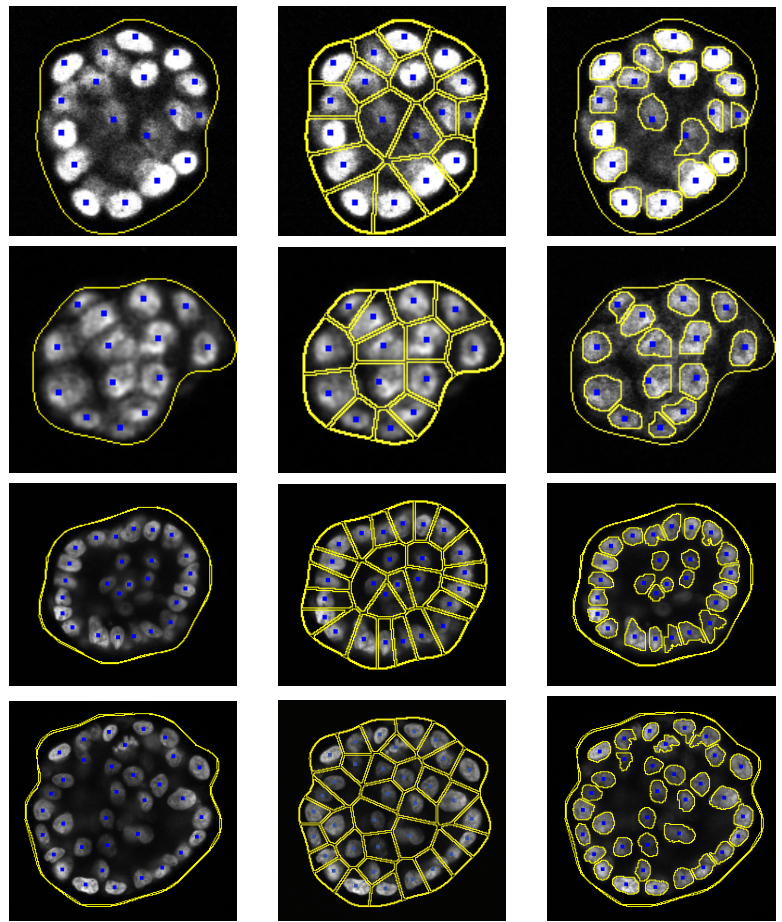

(a)
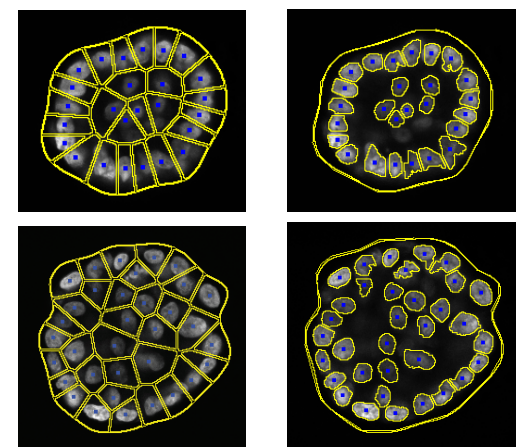

(b)

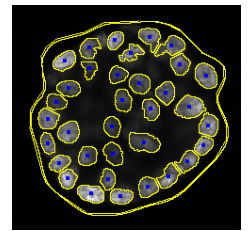

(c)

Fig. 7. Several examples of low and high quality data: (a) The voting results; (b) corresponding Voronoi tessellation; and (c) final segmentation through level set method.

can be generated through iterative spatial voting. The voted landscape can then be used to establish a local neighborhood through Voronoi tessellation where the active contour model delineates nuclear regions from their immediate background. The technique has been tested against 74 images and the performance of the proposed method has been reported. Finally, the method has been applied to a biological problem of interest, and an index for colony organization has been reported that tracks well with the qualitative evaluation of the data collected for this experiment.

\section{REFERENCES}

[1] V. Caseless, R. Kimmel, and G. Sapiro. Geodesic active contours. International Journal of Computer Vision, 22(1):61-79, 1997.

[2] T. Chan and L. Vese. Active contours without edges. IEEE Transactions on Image Processing, 10(2):266-277, 2001.

[3] B. Parvin, Q. Yang, J. Han, H. Chang, B Rydberg, and M. H. Barcellos-Hoff. Iterative voting for inference of structural saliency and characterization of subcellular events. IEEE Transactions on Image Processing, March 2007.

[4] S. Raman, C. A. Maxwell, M. H. Barcellos-Hoff, and B. Parvin. Geometric approach to segmentation and protein localization in cell culture assays. Journal of Microscopy, 225:22-30, 2007.

[5] V.M. Weaver, A.H. Fischer, O.W. Petersen, and M.J. Bissel. The importance of the microenvironment in breast cancer progression: recapitulation of mammary tumorigenesis using a unique human mammary epithelial cell model and a three-dimensional culture assay. Biochemical Cell Biology, 74(12):833-51, 1996.

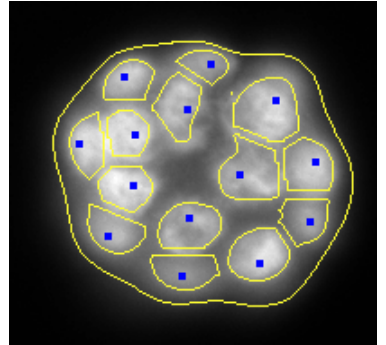

(a)

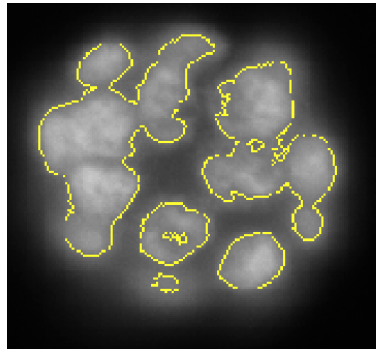

(b)
Fig. 8. Comparison of segmentation in the absence of a local neighborhood established through Voronoi tessellation: (a) nuclear segmentation with the proposed method; and (b) segmentation results with only the level set method applied within the colony.

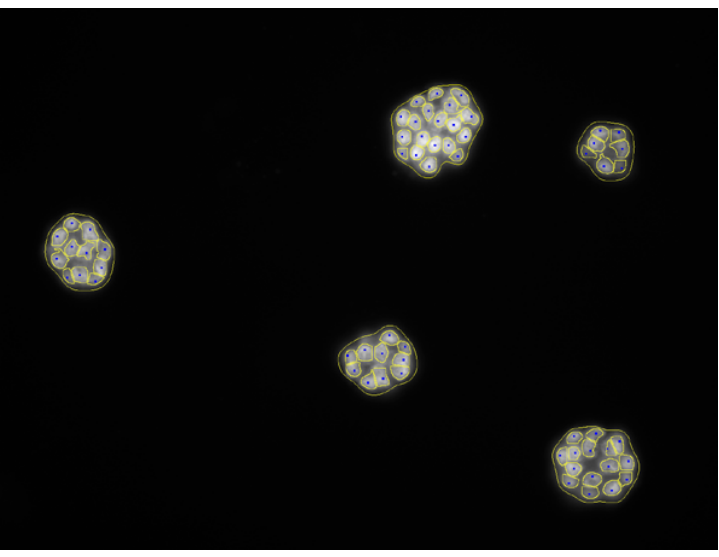

Fig. 9. Multi-colony detection and delineation of each nucleus within the colony.

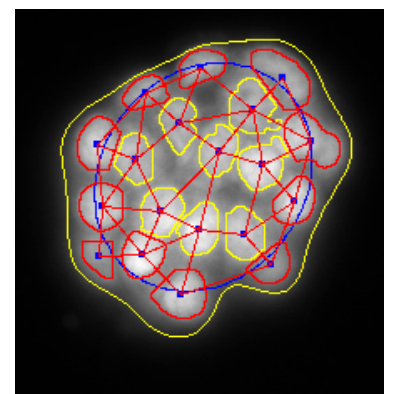

(a)

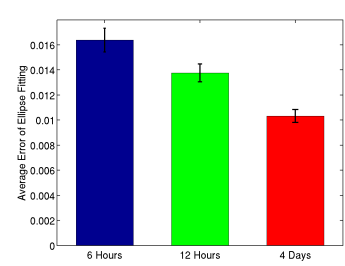

(b)
Fig. 10. (a) Organization of the colony represented as deviation from an ellipse fit; and (b) Change in the computed organization index indicates that colony becomes more elliptical as a function of time after an initial treatment. 